\title{
Holographic Wilson loops in spacetimes with spatial anisotropy
}

\author{
Dmitry S. Ageev, \\ Steklov Mathematical Institute, Russian Academy of Sciences, \\ Gubkina str. 8, 119991, Moscow, Russia \\ E-mail: ageev@mi.ras.ru
}

Irina Ya. Aref'eva

Steklov Mathematical Institute, Russian Academy of Sciences,

Gubkina str. 8, 119991, Moscow, Russia

E-mail: arefevaemi.ras.ru

\section{Anastasia A. Golubtsova*}

The Joint Institute for Nuclear Research,

141980 Dubna, Moscow region, Russia

E-mail: golubtsovaltheor.jinr.ru

In this note we study Wilson loops in an anisotropic black brane background within the holographic framework. The anisotropy is controlled by the so called critical exponent. We calculate time-, space- and light-like static Wilson loops for different orientations of rectangular contours. The first two cases allow us to estimate behaviours of potential and pseudopotential of interquark interaction. We observe that the potential has a similar behaviour comparing to the potential for the isotropic (AdS) case, while the pseudopotential for a certain configuration of the contour has screened Coulomb and linear parts. For the light-like case we derive an analytic expression for the Nambo-Goto action for arbitrary values of the critical exponent.

Corfu Summer Institute 2016 "School and Workshops on Elementary Particle Physics and Gravity" 31 August - 23 September, 2016

Corfu, Greece

* Speaker. 


\section{Introduction}

Wilson loops are fundamental probes of QCD, which allows us to calculate many important quantities, like the potential of interquark interaction, from its expectation values. At the same time, expectation values of static Wilson loops are useful to characterize properties of the quark-gluon plasma produced in heavy ion collisions. Owing to Wilson loops one can perform the analysis of jet quenching, quarkonium suppression, etc.[1,2]. The gauge/gravity duality provides a possibility to consider observables, in particularly Wilson loops, of a gauge theory, dealing with its gravity holographic dual.

In the recent paper [3], we studied Wilson loops in the backgrounds with spatial anisotropy within the holographic framework. In particularly, the evolution of Wilson loops was considered in the time-dependent case to investigate the thermalization process during heavy ion collisions. There is an anisotropy related with the direction along the beam-line in heavy ion collisions. The anisotropic black brane spacetimes and its generalization to the Vaidya solution, that, in our opinion, can serve as the dual description of heavy-ions collision, were found in [4]. The metrics have an anisotropy between spatial directions, which is controlled by the so-called critical exponent. First attempts of using these backgrounds as holographic dual were presented in $[5,6]$. The main motivation for consideration of observables in these backgrounds comes from the fact, that the holographic calculation of the total multiplicity of charged particles production in heavy ions collision reproduces the correct experimental data [7]. At the same time the quark-gluon plasma created in heavy-ion collisions is known to be anisotropic at its early stage of evolutions $[8,9]$ and the holographic model in the anisotropic background is convenient to incorporate anisotropic properties of the QGP.

In the present contribution we calculate holographically Wilson loops in black brane backgrounds with spatial anisotropy [4]. We restrict ourselves by studies of static configurations located on time-like, space-like and light-like contours with different orientations in the spacetime. The time-like and space-like cases represent an extension of our work [3].

In section 2, we review the anisotropic black brane background and the notions of Wilson loops in the standard and holographic prescriptions. In section 3, we consider holographic time-like and space-like Wilson loops for different orientations of the orthogonal contours in the anisotropic backgrounds and corresponding potentials and pseudopotentials of quark-antiquark interactions. In section 4 we calculate Wilson loops for a light-like anisotropic contour in the gravity holographic dual.

\section{Set up}

We start from the gravitational theory given by the action [4]

$$
S=\int d^{5} x \sqrt{|g|}\left(R[g]+\Lambda-\frac{1}{2}(\partial \phi)^{2}-\frac{1}{4} e^{\lambda \phi} F_{(2)}^{2}\right),
$$

where $\phi$ is the dilaton field, $F_{(2)}$ is $U(1)$ gauge field, $\lambda$ is a dilaton coupling constant, and $\Lambda$ is the negative cosmological constant. The corresponding Einstein equations of motion are

$$
R_{m n}=-\frac{\Lambda}{3} g_{m n}+\frac{1}{2}\left(\partial_{m} \phi\right)\left(\partial_{n} \phi\right)+\frac{1}{2} e^{\lambda \phi} F_{m p} F_{n}^{p}-\frac{1}{12} e^{\lambda \phi} F_{(2)}^{2} g_{m n}
$$


The scalar field equation and Maxwell's equation read

$$
\begin{gathered}
\square \phi=\frac{1}{4} \lambda e^{\lambda \phi} F_{(2)}^{2}, \text { with } \square \phi=\frac{1}{\sqrt{|g|}} \partial_{m}\left(g^{m n} \sqrt{|g|} \partial_{n} \phi\right), \\
D_{m}\left(e^{\lambda \phi} F^{m n}\right)=0 .
\end{gathered}
$$

We will consider time-like Wilson loops in gravity backgrounds that are asymptotic to the $5 \mathrm{~d}$ Lifshitz-like geometries [5, 6]

$$
d s^{2}=2 \pi \alpha^{\prime}\left(\frac{-d t^{2}+d x_{1}^{2}}{z^{2}}+\frac{d x_{2}^{2}+d x_{3}^{2}}{z^{2 / v}}+\frac{d z^{2}}{z^{2}}\right)
$$

where $v$ is the critical exponent ${ }^{1}$. We note that the metric (2.5) has boost-invariance, which allows us to construct a shock-wave solutions in [7]. The dilaton and gauge fields are given by

$$
\begin{aligned}
\phi & =\phi(r), \quad e^{\lambda \phi}=\mu e^{4 r}, \\
F_{(2)} & =\frac{1}{2} q d y_{1} \wedge d y_{2},
\end{aligned}
$$

where $q$ and $\mu$ are constants. One can see that the background (2.5) with $v=1$ comes to be the 5-dimensional AdS spacetime.

The direction $x_{1}$ will be referred as a longitudinal one, while the $x_{2}$ and $x_{3}$ are the transverse directions

$$
x_{1}=x_{\|}, \quad x_{2}=x_{3}=x_{\perp} .
$$

The non-zero temperature generalization of (2.5) was found in [4] and can be represented in the following form

$$
d s^{2}=2 \pi \alpha^{\prime}\left(\frac{-f(z) d t^{2}+d x_{1}^{2}}{z^{2}}+\frac{d x_{2}^{2}+d x_{3}^{2}}{z^{2 / v}}+\frac{d z^{2}}{f(z) z^{2}}\right)
$$

with the blackening factor given by

$$
f=1-m z^{2+2 / v}
$$

One can see that for $v=1$ the background (2.9)-(2.10) is the metric of the black brane in the $5 \mathrm{~d}$ AdS spacetime. We note that the values of the parameters $\Lambda, q, \mu, \lambda$ are controlled by the critical exponent. For example, for $v=4$ to satisfy the equations of motion, we have:

$$
\Lambda=90, \quad \mu q^{2}=240, \quad \lambda= \pm \frac{2}{3}
$$

The background (2.9) describes holographically the anisotropic media on the boundary with the temperature corresponding to the Hawking temperature of the black brane:

$$
T=\frac{1}{\pi} \frac{v+1}{2 v} m^{\frac{v}{2 v+2}}
$$

\footnotetext{
${ }^{1}$ Note, that in (2.5) to keep the standard dimension for the metric we put the factor $2 \pi \alpha^{\prime}$ in front of the metric and we assume that all lengths in different directions are dimensionless $\left[\ell_{z}\right]=\left[\ell_{x}\right]=\left[\ell_{y_{1}}\right]=\left[\ell_{y_{2}}\right]=\left(\alpha^{\prime}\right)^{0}$.
} 
In this work we focus on holographic rectangular Wilson loops. The basic formula for the computation of the expectation value of the Wilson loop operator from holography $[11,12]$ (specified by the contour $C$ ) reads as:

$$
W[C]=\left\langle\operatorname{Tr}_{F} e^{i \oint_{C} d x_{\mu} A_{\mu}}\right\rangle=e^{-S_{\text {string }}[C]}
$$

where $S_{\text {string }}[C]$ is the Nambu-Goto action which can be represented as

$$
S_{\text {string }}=\frac{1}{2 \pi \alpha^{\prime}} \int d \tau d \sigma \sqrt{-\operatorname{det} h_{\alpha \beta}}
$$

with the induced metric of the world-sheet

$$
h_{\alpha \beta}=g_{M N} \partial_{\alpha} X^{M} \partial_{\beta} X^{N}
$$

where $\alpha, \beta=1,2$.

The potential of the interquark interaction can be extracted from the rectangular time-like Wilson loop of size $T \times X$, i.e. the loop in which one side is infinite along the time direction, and the other is along the spatial one,

$$
W(T, X)=\left\langle\operatorname{Tr} e^{i \oint_{T \times X} d x_{\mu} A_{\mu}}\right\rangle \sim e^{-V(X) T}
$$

A similar operator to probe QCD, the spatial rectangular Wilson loop of size $X \times Y$,defines the so called pseudopotential $\mathscr{V}$, is given by

$$
W(X, Y)=\left\langle\operatorname{Tr} e^{i \oint_{X \times Y} d x_{\mu} A_{\mu}}\right\rangle=e^{-\mathscr{V}(X) Y} .
$$

The pseudopotential can be straightforwardly extracted from the string action as follows

$$
\mathscr{V}(X)=\frac{S_{\text {string }}}{Y}
$$

As it is known from the QCD lattice calculations the spatial Wilson loops obey the area law at all temperature, i.e.

$$
\mathscr{V}(X) \sim \sigma_{s} X
$$

where $\sigma_{s}$ defines the spatial string tension

$$
\sigma_{s}=\lim _{X \rightarrow \infty} \frac{\mathscr{V}(X)}{X}
$$

The quantity $\sigma_{s}$ differs from the usual string tension which is defined from time-like Wilsonloops. By virtue to the non-Abelian Stokes formula equal time spatial Wilson loops [14] are related with the spatial components of the energy-momentum tensor and by this reason $\sigma_{s}$ is also called the magnetic string tension. 


\section{Holographic Wilson loops in spacetimes with spatial anisotropy}

\subsection{Holographic time-like Wilson loops}

Here we consider two cases of the orientation of time-like Wilson loops. For both configurations we suppose that the string is stretched in the radial direction, so $z=z(\sigma)$, as well as we assume that the strip is infinite along the time direction.

We start with the loop located on the $t x_{\|}$-plane. We take the parametrization $\tau=t, \sigma=x_{\|}$. Thus, the Nambo-Goto action (2.14) for this case reads

$$
S=\frac{1}{2 \pi \alpha^{\prime}} \int d \sigma \frac{\sqrt{\left(f(z)+z^{\prime 2}\right)}}{z^{2}} .
$$

The equation of motion corresponding to (3.1) is given by

$$
z^{\prime}= \pm \sqrt{f(z)\left(\frac{f(z)}{z^{4} \mathscr{I}^{2}}-1\right)}
$$

with the following integral

$$
\mathscr{I}=-\frac{f(z)}{z^{2} \sqrt{f(z)+z^{\prime 2}}} .
$$

The length of the two endpoints of the string on the brane can be calculated from (3.2)

$$
\ell=2 \int_{z_{0}}^{z_{*}} d z \sqrt{\frac{f\left(z_{*}\right) z^{4}}{f(z)\left(z_{*}^{4} f(z)-f\left(z_{*}\right) z^{4}\right)}},
$$

where the integral of motion is related to the turning point, for which $z^{\prime}=0$, as follows

$$
\mathscr{I}^{2}=\frac{f\left(z_{*}\right)}{z_{*}^{4}} .
$$

Performing the change of variables in (3.1) and taking into to the relation for $z^{\prime}$ (3.2) one obtains

$$
S_{x_{\|}, t_{(\infty)}}=T \int d z \sqrt{\frac{f(z) z_{*}^{4}}{z^{4}\left[f(z) z_{*}^{4}-f\left(z_{*}\right) z^{4}\right]}}
$$

Performing the renormalization by subtraction the mass of the two free quark $[8,12,13]$ we get for the energy of the string between two quarks stretched in the longitudinal direction

$$
E_{x_{\|}, t_{(\infty)}, r e n}=\int_{0}^{z_{*}} \frac{d z}{z^{2}}\left[\sqrt{\frac{f(z) z_{*}^{4}}{f(z) z_{*}^{4}-f\left(z_{*}\right) z^{4}}}-1\right]-\frac{1}{z_{*}}+\frac{1}{z_{h}},
$$

taking into account that $z_{h}=\frac{1}{m^{\frac{1}{2+2 / v}}}$, we get that the energy of two free quarks that has to be subtracted is equal to $m^{\frac{1}{2+2 / v}}$.

In Fig.1 (a) we present the dependence of the energy (3.7) on the length (3.4). We observe that the essential dependence on the anisotropic parameter $v$ appears with growing values of the distance between the quark and the anti-quark. One should be noted that for enough small $\ell$ the 


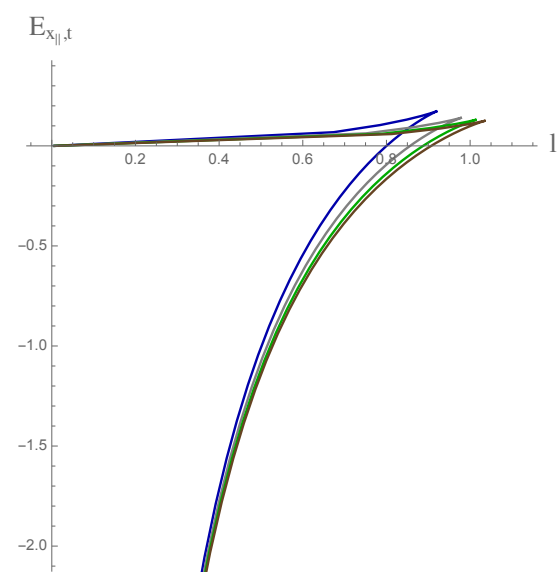

(a)

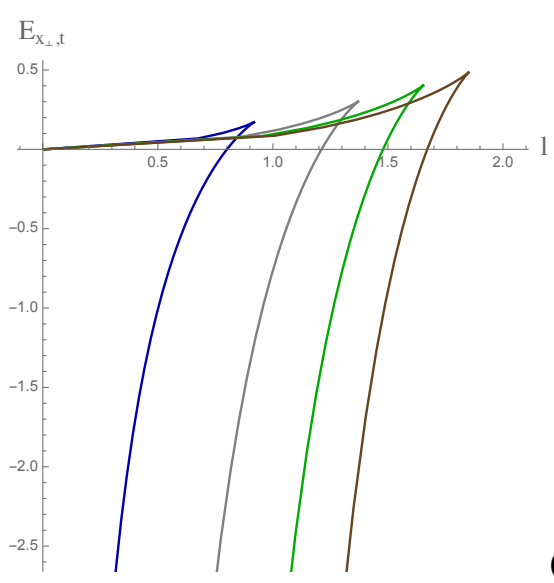

(b)

Figure 1: (a).The string energy (3.7) as a function of the interquark distance (3.4) for $v=1,2,3,4$ (from top to bottom, respectively). (b). The string energy (3.14) stretched in the transversal direction as a function of the interquark distance (3.11) for $v=1,2,3,4$ (from left to right, respectively).

behaviour of the string energy extracted from (3.7) for all $v$ is close to the behaviour in the case $v=1$ (the AdS case).

Now we turn to the configuration on the $t x_{\perp}$-plane, so we take the parametrization $\sigma=x_{\perp}$. The Nambo-Goto action (2.14) for this case is

$$
S=\frac{1}{2 \pi \alpha^{\prime}} \int d \sigma \frac{\sqrt{f(z)+z^{2 / v-2} z^{\prime 2}}}{z^{1+1 / v}} .
$$

The equation of motion following from (3.8) reads

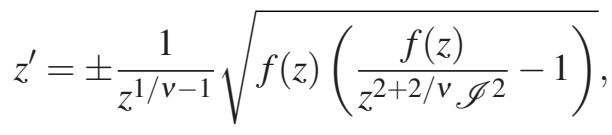

with the integral of motion is given by

$$
\mathscr{I}=\frac{z^{-1-1 / v} f(z)}{\sqrt{f(z)+z^{2 / v-2} z^{\prime 2}}} .
$$

The distance between two quarks can be calculated from (3.9) as

$$
\ell_{x_{\perp}}=2 \int_{z_{0}}^{z_{*}} d z z^{1-1 / v} \sqrt{\frac{f\left(z_{*}\right) z^{2+2 / v}}{f(z)\left(z_{*}^{2+2 / v} f(z)-f\left(z_{*}\right) z^{2+2 / v}\right)}},
$$

the relation between the integral of motion $\mathscr{I}$ and the turning point corresponding to (3.10) is

$$
\mathscr{I}^{2}=\frac{f\left(z_{*}\right)}{z_{*}^{2+2 / v}} .
$$

Plugging (3.10) into (3.8) and coming to the integration with respect to $z$-variable one gets 


$$
S_{t, x_{\perp}}=T \int_{z_{0}}^{z_{*}} d z \frac{1}{z^{2}} \sqrt{\frac{z_{*}^{2+2 / v} f(z)}{z_{*}^{2+2 / v} f(z)-f\left(z_{*}\right) z^{2 / v+2}}} .
$$

Taking into account the relation for the blackening function and performing renormalization, for the energy of the string between two quarks stretched in the transversal direction one obtains

$$
E_{x_{\perp} t_{\infty}, r e n}=\int_{z_{0}}^{z_{*}} \frac{d z}{z^{2}}\left[\sqrt{\frac{z_{*}^{2+2 / v} f(z)}{z_{*}^{2+2 / v} f(z)-f\left(z_{*}\right) z^{2+2 / v}}}-1\right]-\frac{1}{z_{*}}+\frac{1}{z_{h}},
$$

where $z_{h}=\frac{1}{m^{2+2 / v}}$.

Fig.1 (b) we present the dependence of the string energy (3.14) on the length (3.11). One can see that the dependence of $E_{x_{\perp} t_{\infty}, r e n}$ on the critical exponent disappears at large $\ell$ and for all $v$ are linear growing functions slightly deviating from the AdS case $(v=1)$. We also see that the energy is minimal (on the absolute value) for the isotropic configuration.

Comparing Fig.1 (a) and Fig.1 (b) one can say that the form of the string energy is similar to the AdS case. This means that the dual gauge theory should be a continuous deformation of $\mathscr{N}=4$ sYM.

\subsection{Holographic spatial Wilson loops}

In this section we will consider spatial Wilson loops located in the planes $x_{1} x_{2}$ and $x_{2} x_{3}$.

Let us start with a rectangular Wilson loop in the $x_{1} x_{2}$ plane with a short side of the length $\ell$ in the longitudinal $x$ direction and a long side of the length $L_{x_{2}}$ along the transversal $x_{2}$ direction, so that

$$
x_{1} \in\left[0, \ell<L_{x_{1}}\right], \quad x_{2} \in\left[0, L_{x_{2}}\right] .
$$

We assume that the Wilson loop is invariant under the $x_{2}$-direction. We parameterize the world-sheet of the string in the following way $\sigma^{1}=x_{1}, \sigma^{2}=x_{2}$.

The Nambo-Goto action (2.14) takes the form

$$
S_{x_{1}, x_{2(\infty)}}=L_{x_{2}} \int_{z_{0}}^{z_{*}} \frac{1}{z^{1+1 / v}} \frac{d z}{\sqrt{f(z)\left(1-\left(\frac{z}{z_{*}}\right)^{2+2 / v}\right)}}
$$

where $z_{*}$ is the turning point.

The distance between the quark and the anti-quark can be rewritten in terms of the dimensionless variable $w=z / z_{*}$ as

$$
\ell=2 z_{*} \int_{z_{0} / z_{*}}^{1} \frac{w^{1+1 / v} d w}{\sqrt{f\left(z_{*} w\right)\left(1-w^{2+2 / v}\right)}},
$$

while the renormalized Nambu-Goto action (3.16) in terms of $w$ is represented as

$$
S_{x_{1}, x_{2(\infty)}, r e n}=L_{x_{2}}\left[\frac{1}{z_{*}^{1 / v}} \int_{0}^{1} \frac{d w}{w^{1+1 / v}}\left[\frac{1}{\sqrt{f\left(z_{*} w\right)\left(1-w^{2+2 / v}\right)}}-1\right]-\frac{v}{z_{*}^{1 / v}}\right] .
$$


Then pseudopotential $\mathscr{V}_{x_{1}, x_{2(\infty)}}$ is given by:

$$
\mathscr{V}_{x_{1}, x_{2(\infty)}}=\frac{S_{x_{1}, x_{2(\infty)}, r e n}}{L_{x_{2}}}
$$

In Fig.2 (a) we present the dependence of the pseudopotential $\mathscr{V}_{x_{1}, x_{2}(\infty)}$ (3.19) on the length (3.17). We observe that for small $\ell$ the pseudopotential has the Coulomb part deformed by the critical exponent, thus

$$
\mathscr{V}_{x_{1}, x_{2(\text { infty })}}(\ell, v) \sim-\frac{\mathscr{C}_{1}(v)}{\ell^{1 / v}}, \quad \ell_{x_{1}} \rightarrow 0,
$$

with some constant $\mathscr{C}_{1}$ dependent on $v$. For large $\ell$ the pseudopotential $\mathscr{V}$ behaves as a linearly increasing function

$$
\mathscr{V}_{x_{1}, x_{2(\text { infty) }}}(\ell, v) \sim \sigma_{s, 1}(v) \ell, \quad \ell_{x_{1}} \rightarrow \infty .
$$

Now we come to a rectangular loop in the $x_{1} x_{2}$-plane with a short side of the length $\ell$ in the $x_{2}$ direction and a long side of the length $L_{x_{1}}$ along the $x_{1}$-direction,

$$
x_{1} \in\left[0, L_{x_{1}}\right], \quad x_{2} \in\left[0, \ell<L_{x_{2}}\right] .
$$

The distance between two quarks can be written down in terms of the $w$-variable

$$
\ell=2 z_{*}^{1 / v} \int_{0}^{1} \frac{w^{2 / v} d w}{\sqrt{f\left(z_{*} w\right)\left(1-w^{2+2 / v}\right)}}
$$

where the $z_{*}$ is the turning point.

The renormalized Nambu-Goto action (3.23) reads

$$
S_{x_{2}, x_{1,(\infty)}, r e n}=L_{x_{1}}\left[\frac{1}{z_{*}} \int_{z_{0} / z_{*}}^{1} \frac{d w}{w^{2}}\left[\frac{1}{\sqrt{f\left(z_{*} w\right)\left(1-w^{2+2 / v}\right)}}-1\right]-\frac{1}{z_{*}}\right],
$$

while the pseudopotential $\mathscr{V}_{x_{2}, x_{1,(\infty)}}$ related to (3.24) is:

$$
\mathscr{V}_{x_{2}, x_{1,(\infty)}}=\frac{S_{x_{2}, x_{1,(\infty)}, r e n}}{L_{x_{1}}} .
$$

In Fig. 2 (b) we show the dependence of the pseudopotential extracted from the action (3.24) on the length $\ell$ for different values of the temperature and the dynamical exponent. The pseudopotential has a power-law dependence on $v$ for small $\ell_{x_{2}}$, so that

$$
\mathscr{V}_{x_{2}, x_{1,(\infty)}} \sim-\frac{\mathscr{C}_{2}(v)}{\ell_{x_{2}}^{v}}, \quad \ell_{x_{2}} \rightarrow 0
$$

with some constant $\mathscr{C}_{2}$ dependent on $v$.

For large distances the pseudopotential represents a linear function of $\ell$ again

$$
\mathscr{V}_{x_{2}, x_{1(\infty)}}\left(\ell_{x_{2}}, v\right) \sim \sigma_{s, 2}(v) \ell_{x_{2}}, \quad \ell_{x_{2}} \rightarrow \infty
$$


Finally, we turn to the Wilson loop located in the transversal $x_{2} x_{3}$-plane with a short side of the length $\ell$ in one of transversal directions and a long side of the length $L_{x_{3}}$ along the other transversal direction, namely

$$
x_{2} \in\left[0, \ell<L_{x_{2}}\right], \quad x_{3} \in\left[0, L_{x_{3}}\right] .
$$

We choose only transversal coordinates for the parameterization of the worldsheet $\sigma^{1}=x_{2}, \sigma^{2}=$ $x_{3}$.

The relation for the length in terms of the $w$-variable reads

$$
\ell=z_{*}^{1 / v} \int \frac{d w}{w^{1-3 / v} \sqrt{f\left(z_{*} w\right)\left(1-w^{4 / v}\right)}}
$$

where $z_{*}$ is the turning point.

The renormalized Nambo-Goto action takes the form

$$
S_{x_{2}, x_{3(\infty)}, r e n}=L_{x_{3}}\left(\frac{1}{z_{*}^{1 / v}} \int_{z_{0} / z_{*}}^{1} \frac{d w}{w^{1+1 / v}}\left[\frac{1}{\sqrt{f\left(z_{*} w\right)\left(1-w^{4 / v}\right)}}-1\right]-\frac{v}{z_{*}^{1 / v}}\right) .
$$

The pseudopotential $\mathscr{V}_{x_{2}, x_{3(\infty)}}$ extracted from (3.30) can be represented as:

$$
\mathscr{V}_{x_{2}, x_{3(\infty)}}=\frac{S_{x_{2}, x_{3(\infty)}}}{L_{x_{3}}}
$$

In Fig.2 (c) we display the behaviour of the pseudopotential (3.31) on the length (3.29).

One can see that the behavior of $\mathscr{V}_{x_{2}, x_{3(\infty)}}$ in Fig.2(c) is rather different from two previous cases. We observe, that the dependence on $v$ is driven by some constant $\mathscr{C}_{3}$ relying on $v$. It should be noted that the pseudopotentials strongly deviate from the $A d S$ case $(v=1)$ both in the UV and the IR regions of $\ell$. Thus, one can write for small $\ell$

$$
\mathscr{V}_{x_{2}, x_{3(\infty)}}\left(\ell_{x_{2}}, v\right) \sim-\frac{\mathscr{C}_{3}(v)}{\ell_{x_{2}}}, \quad \ell_{x_{2}} \rightarrow 0
$$

with some constant $\mathscr{C}_{3}$ dependent on $v$.

At the same time for large $\ell$ we have

$$
\mathscr{V}_{x_{2}, x_{3(\infty)}}\left(\ell_{x_{2}}, v\right) \sim \sigma_{s, 3}(v) \ell_{x_{2}}, \quad \ell_{x_{2}} \rightarrow \infty
$$

\section{Holographic light-like Wilson loops}

It is well known that light-like Wilson loops are related with jet quenching parameter, that described by a ratio of mean transverse momentum obtained by the parton moving through the plasma over the travelled distance. Its holographic calculation in different backgrounds has been considered in [15]-[21]. 


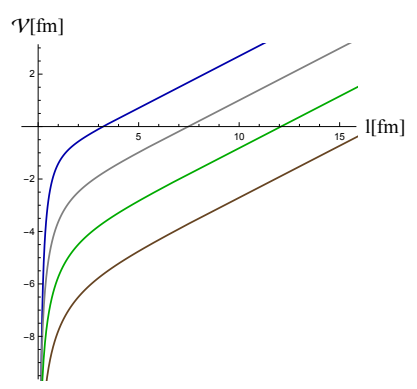

(a)
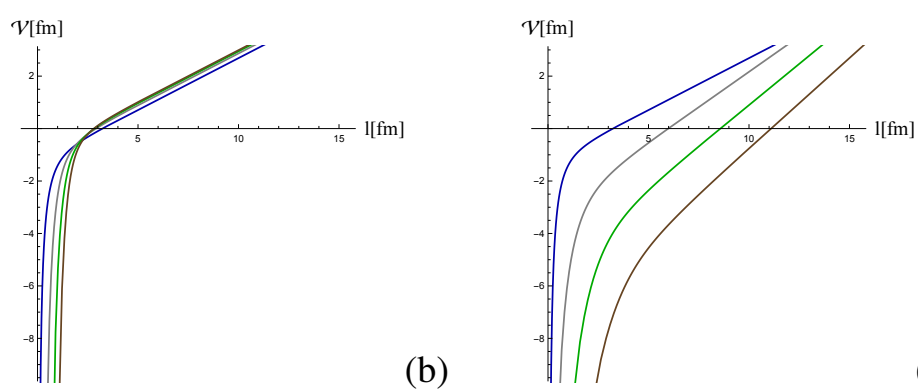

(c)

Figure 2: (a),(b),(c):The behaviours of the pseudopotentials $\mathscr{V}_{x_{1}, x_{2(\infty)}}, \mathscr{V}_{x_{2}, x_{1,(\infty)}}, \mathscr{V}_{x_{2}, x_{3(\infty)}}$, correspondingly, for $v=1,2,3,4$ (from top to down) as functions of the distance between the quarks $\ell$ at $T=0.2$.

To calculate the holographic light-like Wilson loop we perform the coordinate transformation $x^{ \pm}=\frac{t \pm x_{1}}{\sqrt{2}}$, then the metric in light-cone coordinates takes the form

$$
d s^{2}=-\frac{(1+f(z))}{z^{2}} d x^{+} x^{-}+\frac{1-f(z)}{2 z^{2}}\left[d x^{+2}+d x^{-2}\right]+\frac{d x_{2}^{2}}{z^{2 / v}}+\frac{d x_{3}^{2}}{z^{2 / v}}+\frac{d z_{2}^{2}}{z^{2}} .
$$

We choose the ansatz for the string configuration

$$
\sigma^{1}=x^{-}, \quad \sigma^{2}=x^{+}, \quad z=z\left(\sigma^{2}\right) .
$$

We suppose that the string worldsheet has translational invariance along $x_{1}$. The Nambo-Goto action then reads

$$
S_{x^{-} x_{1}}=\frac{L^{-} \sqrt{m}}{\sqrt{2} \pi \alpha^{\prime}} \int_{0}^{\ell / 2} d x_{1} \sqrt{1+\frac{z^{\prime 2}}{z^{2-2 / v} f}} .
$$

with $z^{\prime}=d z / d x_{1}$. The integral of motion corresponding to (4.3) reads

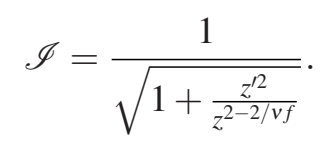

The equation of motion reads

$$
z^{\prime}=\frac{\sqrt{1-\mathscr{I}^{2}}}{\mathscr{I}} z^{1-1 / v} \sqrt{1-m z^{2+2 / v}}
$$

Plugging (4.5) into (4.3) we have

$$
S_{x^{-} x_{1}}=\sqrt{m} \frac{L^{-}}{\mathscr{I} \sqrt{2} \pi \alpha^{\prime}} \ell
$$

while the length of the string reads

$$
\ell=\frac{\mathscr{I}}{\sqrt{1-\mathscr{I}^{2}}} \int_{0}^{z_{*}} \frac{d z}{z^{1-1 / v} \sqrt{1-m z^{2+2 / v}}} .
$$

We consider the situation when the turning point $z_{*}=z_{h}=m^{-\frac{1}{2+2 / v}}$. Then one obtains

$$
\ell=-\frac{\mathscr{I}}{\sqrt{1-\mathscr{I}^{2}}} m^{-\frac{1}{2(v+1)}} \frac{\sqrt{\pi} \Gamma\left(\frac{1}{2+2 v}\right)}{\Gamma\left(-\frac{v}{2+2 v}\right)},
$$


or

$$
\ell=-a_{v}(m) \frac{\mathscr{I}}{\sqrt{1-\mathscr{I}^{2}}}, \quad \text { with } \quad a_{v}(m)=m^{-\frac{1}{2(v+1)}} \frac{\sqrt{\pi} \Gamma\left(\frac{1}{2+2 v}\right)}{\Gamma\left(-\frac{v}{2+2 v}\right)} .
$$

Taking into account (2.12) we can write

$$
a_{v}(m)=-T^{-1 / v} a_{T, v}, \quad \text { with } \quad a_{T, v}=\left(\frac{2 \pi v}{v+1}\right)^{-1 / v} \frac{\sqrt{\pi} \Gamma\left(\frac{1}{2+2 v}\right)}{\Gamma\left(-\frac{v}{2+2 v}\right)}, \quad T=\frac{1}{\pi} \frac{(v+1)}{2 v} m^{\frac{v}{2 v+2}} .
$$

Owing to (4.10) the string action with subtracted the divergences reads

$$
\Delta S_{x^{-} x_{1}}=\left(\frac{2 \pi v}{v+1}\right)^{1+1 / v} \frac{L^{-} T a_{T, v}}{\sqrt{2} \pi \alpha^{\prime}}\left[\sqrt{1+\frac{T^{2 / v} \ell^{2}}{a_{T, v}^{2}}}-1\right]
$$

\section{Conclusions}

In this short contributions we have considered Wilson loops in an anisotropic holographic gravity dual. The black brane background has a spatial anisotropy and motivated by the possibility to reproduce the dependence of the multiplicity of particles created in heavy-ion collisions on the energy using the holographic duality. We have discussed static configurations located on time-like, space-like and light-like rectangular contours with different orientation in the spacetime.

We observe that the behavior of the potential of an interquark interaction as a function of the distance between two quarks is similar to the isotropic case. So the dual gauge theory is supposed to be a deformation of $\mathscr{N}=4$ SYM theory. From the form of the potential one can also conclude that there is no confinement in the holographic model. However, looking at the behavior of the pseudopotential corresponding to the configuration on the transversal plane one can see that it has the screened Cornell form. We have also calculated the light-like Wilson loop and derive the analytic expression for the Nambo-Goto action for an arbitrary value of the critical exponent.

A possible extension of this work is to more detailed study the jet quenching. It will also be interesting to deal with metrics providing the confinement, compare [22], and to perform full study of the observables using the holographic approach.

Acknowledgements AG would like to thank the organizers of the Corfu Summer Institute and the Workshop on Recent Developments in Strings and Gravity for kind hospitality and the opportunity to present this work.

\section{References}

[1] J. Casalderrey-Solana, H. Liu, D. Mateos, K. Rajagopal, U. A. Wiedemann, Gauge/String Duality, Hot QCD and Heavy-Ion Collisions,[arXiv:1101.0618].

[2] I. Ya. Aref'eva, "Holographic approach to quark-gluon plasma in heavy ion collisions,' Phys. Usp. 57, 527 (2014) [Usp. Fiz. Nauk 184, no. 6, 569 (2014)];

[3] D.S. Ageev, I. Ya. Aref'eva, A. A. Golubtsova and E. Gourgoulhon, Holographic Wilson loops in Lifshitz-like backgrounds, [arXiv:1606.03995]. 
[4] I. Y. Aref'eva, A. A. Golubtsova and E. Gourgoulhon, Analytic black branes in Lifshitz-like backgrounds and thermalization, JHEP 09 (2016) 142; [arXiv: 1601.06046 ].

[5] M. Taylor, Non-relativistic holography; [arXiv:0812.0530].

[6] T. Azeyanagi, W. Li and T. Takayanagi, On string theory duals of Lifshitz-like fixed points, JHEP 06 (2009) 084; [arXiv:0905.0688].

[7] I. Ya. Aref'eva and A. A. Golubtsova, Shock waves in Lifshitz-like spacetimes, JHEP 04 (2015) 011; [arXiv:1410.4595].

[8] D. Giataganas, Probing strongly coupled anisotropic plasma, JHEP 07 (2012) 031; [arXiv:1202.4436].

[9] M. Strickland, Thermalization and isotropization in heavy-ion collisions, Pramana 84, 671 (2015); [arXiv:1312.2285].

[10] I. Ya. Aref'eva, Formation time of quark-gluon plasma in heavy-ion collisions in the holographic shock wave model, Theoret. and Math. Phys., 184 (2015), 1239-1255; [arXiv: 1503.02185 ].

[11] J. M. Maldacena, Wilson loops in large N field theories, Phys.Rev.Lett. 80(1998) 4859-4862; [arXiv:hep-th/9803002].

[12] S. J. Rey, S. Theisen and J. T. Yee, Wilson-Polyakov loop at finite temperature in large N gauge theory and anti-de Sitter supergravity, Nucl. Phys. B 527, 171 (1998); [arXiv: hep-th/9803135].

[13] A. Brandhuber, N. Itzhaki, J. Sonnenschein and S. Yankielowicz, Phys. Lett. B 434 (1998); [arXiv:hep-th/9803137].

[14] I. Arefeva, NonAbelian Stokes formula, Theor. Math. Phys. 43, 353 (1980).

[15] H. Liu, K. Rajagopal and U. A. Wiedemann, "Calculating the jet quenching parameter from AdS/CFT,” Phys. Rev. Lett. 97, 182301 (2006) [arXiv: hep-ph/ 0605178 ].

[16] H. Liu, K. Rajagopal and U. A. Wiedemann, "Wilson loops in heavy ion collisions and their calculation in AdS/CFT,” JHEP 0703, 066 (2007); [arXiv : hep-ph / 0612168 ].

[17] U. Gursoy, E. Kiritsis, L. Mazzanti, G. Michalogiorgakis and F. Nitti, "Improved Holographic QCD," Lect. Notes Phys. 828, 79 (2011); [arXiv: hep-th/1006.5461].

[18] M. Chernicoff, D. Fernandez, D. Mateos and D. Trancanelli, "Jet quenching in a strongly coupled anisotropic plasma," JHEP 1208, 041 (2012); [arXiv: hep-th/1203.0561].

[19] A. Rebhan and D. Steineder, "Probing Two Holographic Models of Strongly Coupled Anisotropic Plasma," JHEP 1208, 020 (2012); [arXiv: hep-th/1205.4684].

[20] D. Giataganas, “Observables in Strongly Coupled Anisotropic Theories,” PoS Corfu 2012, 122 (2013); [arXiv:hep-th/1306.1404].

[21] J. Sadeghi and S. Heshmatian, "Jet Quenching Parameter with Hyperscaling Violation,” Eur. Phys. J. C 74, 3032 (2014); [arXiv: hep-th/1308.5991].

[22] I. Ya. Aref'eva, Holography for Heavy-Ion Collisions at LHC and NICA; [arXiv: 1612. 08928 ]. 